\title{
A Multidisciplinary Look and Its Benefits Associated with the Gut Microbiota
}

\author{
Glauber Pimentel Florêncio', Andréa Barros Ferreira Maiolino ${ }^{2}$, Eliza Helena Eliete Silva ${ }^{3}$, \\ Ralciane de Paula Menezes ${ }^{4}$ and Denise Von Dolinger de Brito ${ }^{5 *}$ \\ ${ }^{1}$ Gynecology and Obstetrics Service, Comprehensive Health Care Center, Brazil \\ ${ }^{2}$ Psychologist, Brazil \\ ${ }^{3}$ Nutritionist, Brazil \\ ${ }^{4}$ Technician at the Technical Health School of the Federal University of Uberlândia, Brazil \\ ${ }^{5}$ Professor of the Postgraduate Program in Health Sciences, Federal University of Uberlândia, Brazil
}

*Corresponding author: Denise Von Dolinger de Brito, Professor of the Postgraduate Program in Health Sciences, Faculty of Medicine, Federal University of Uberlândia, Brazil

To Cite This Article: Glauber Pimentel Florêncio, Andréa Barros Ferreira Maiolino, Eliza Helena Eliete Silva, Ralciane de Paula Menezes, Denise Von Dolinger de Brito. A Multidisciplinary Look and Its Benefits Associated with the Gut Microbiota. Am J Biomed Sci \& Res. 2021 - 13(3). AJBSR. MS.ID.001872. DOI: 10.34297/AJBSR.2021.13.001872.

Received: 眥 June 12, 2021; Published: 制 June 29, 2021

\section{Introduction}

Millions of microorganisms are present in regions of the human body that have access to the external environment, such as the oral cavity, gastrointestinal tract, respiratory tract, skin and sexual organ. Among these microorganisms are bacteria, fungi, viruses and protozoa [1]. The agglomeration of these microorganisms inhabiting a certain region of the body is called microbiota. The microorganisms that specifically inhabit the gastrointestinal tract make up the intestinal microbiota. It is in this environment that the greatest density and diversity of these microorganisms resides, which are mostly bacteria [2]. A healthy gut microbiota is characterized by its resilience, although diversity is also an implicit feature. It is also one that plays important roles in the human body, such as the production of short-chain fatty acids that have immunological, anti-inflammatory and metabolic actions. Certain bacteria have been associated with the production of these metabolites and are therefore considered health promoters [3].

The composition of intestinal bacteria can be modulated by several factors including birth by vaginal delivery and cesarean section, work environment, especially if linked to the health area, exposure to xenobiotics, food, medication, physical activity, stressors, antibiotics, smoking and use of antidepressants. However, allergic processes caused by known inflammatory foods trigger inflammatory processes of a mild to severe nature, from acute to chronic [4]. The inflammatory process can be silent until it reaches the point of having clinical manifestations with signs and symptoms. both through changes in diet, physical activity and the use of medications, food intolerance and obesity. It is known that after birth until adulthood, the composition and activity of the microbiota becomes more complex, in the elderly, for example, the vulnerability of the microbiota is characterized by an inflammatory process that is subject to changes in digestion, absorption and immunity $[5,6]$.

Therefore, it can be seen that the microbiota is susceptible to changes due to several factors, whether environmental or genetic. When there is a qualitative or quantitative change in the microbiota it is called dysbiosis. It causes changes not only in the intestine, but also in almost the entire system of the human body, ranging from autoimmune diseases, obesity and even behavioral or cognitive changes. From in vivo studies in both animals and humans, it was observed that dysbiosis is associated with the growth of pathobionts, which are present in smaller quantities, however, in disturbances in the intestinal ecosystem, these bacteria may proliferate with a potential pathogenic [7]. There is also the partial or loss of commensals that could fight the proliferation of these pathogens and the restoration of these bacteria can reverse dysbiotic factors. In addition, there may be a loss of diversity 
that has been linked to inadequate diets and inflammatory bowel diseases [8].

\section{Healthy Diet}

Diet is the biggest modulating factor of the intestinal microbiota, directly affecting its ecological abundance, diversity and uniformity [9]. Food contains thousands of bioactive molecules that nourish and stimulate the metabolism of intestinal microorganisms, that is, if not balanced, it leads to disturbances in the constitution and function of microbial populations, resulting in the production of inflammatory metabolites that induce cell proliferation and inflammation that, by finally, they increase the risk of neoplasia [10].

There is a complex relationship between diet, bile acids and microbiota. High fat diets are related to increased biliary secretion and risk of colorectal cancer [11] through the following mechanism: primary bile acids produced in the liver from cholesterol are excreted and converted through bacterial metabolism into acids secondary biliary. These are used as an energy source by other members of the microbiota [12], but they are also associated as a mechanism of carcinogenesis of colorectal tumors by interfering with the process of cell proliferation, apoptosis, induction of DNA damage through the production of oxidative radicals, such as ROS and NOS (oxidative radicals and nitrosamines) [13].

Studies show that the dietary pattern has a direct influence on the composition of the microbiota. Dash et al. concluded that dietary change results in rapid phenotypic change and that the results of this change remain, suggesting that long-term dietary changes can have significant and lasting effects on microbiota health [14].

\section{Rural area versus Urban area}

The healthy pattern of the microbiota is related to its diversity, as described in population studies [15]. Such diversity is influenced by various sociocultural and geographical aspects and the rural or urban environment can be one of them. Therefore, researchers conducted a cohort study and observed that there was no taxonomic difference between the composition of the microbiota of inhabitants of urban and rural areas in Russia. However, the rural population had a higher proportion of species not identified in previous studies, which may mean greater microbiota diversity, while urban inhabitants have a microbiota more similar to Western countries people [16].

\section{Physical activity}

Regular practice of moderate physical exercise has numerous health benefits, including a reduction in the risk of obesity and associated comorbidities; the improvement of bone and muscle metabolism; reducing the risk of gastrointestinal diseases such as colon cancer; among others [17]. However, the influence that physical exercise has on intestinal health, and in particular on the intestinal microbiota, is a recent area of investigation, with few studies carried out in humans. In these studies, exercise seems to affect the diversity, composition and some of the functions performed by the intestinal microbiota.

The possibility of correlation between physical activity and intestinal microbiota has been tested by scientists [18]. Davis CD in his research compared germ-free versus colonized mice, both with obesogenic diet, suggest that the manipulation of the microbial community is able to influence the bioenergetic response of the muscles, being protective against the western high-density diet. An intervention study with physical activity showed a correlation with the microbiota [19]. An experiment carried out with germfree mice showed that they had worse physical performance when compared to those colonized with a single filament of bacteria, which, in turn, showed lower endurance capacity when compared to those colonized with several types of non-pathogenic bacteria [20]. In this study, the composition of the microbiota interfered in the athletic performance of mice by altering antioxidant activity.

Studies carried out in humans corroborate these data found in animal models. In fact, it was observed in cross-sectional studies that there are significant differences in the composition of gut microbiota present in feces between trained and sedentary individuals, in which physically active individuals and athletes have greater diversity and species richness compared to sedentary individuals. It is possible to observe this difference through statistical methods that allow analyzing the complexity and diversity of gut microbiota between groups, such as principal coordinate analysis (PCoA) [21,22,23,24]. In addition, aerobic capacity, represented by the maximum oxygen consumption $\left(\mathrm{VO}_{2}\right.$ max) during a test of maximum effort is an important characteristic that distinguishes these trained and sedentary subjects in crosssectional studies. In this sense, Estaki et al demonstrated that there is a positive correlation between $\mathrm{VO}_{2}$ max and species richness of intestinal bacteria [23].

\section{Role of therapy in the care of the intestinal microbiota}

Anxiety is an emotional state where a person finds himself in a period of fear, tension, excessive worry and discomfort. Generalized anxiety disorder (GAD) is a disorder characterized by marked anxiety and worry, and these symptoms chronically compromise the quality of life and other areas of the individual's functional capacity $[25,26]$.

The trillions of microorganisms located in the intestine are called intestinal microbiota, and they play important roles in the immune system and metabolism, providing essential inflammatory mediators, nutrients and vitamins [27]. Furthermore, Toll-like 
receptors (TLR) can specifically recognize lipopolysaccharide (LPS) molecules in pathogenic microorganisms, especially TLR4. After LPS from the gut microbiota activates the TLR, the NF- $\kappa$ B pathway that regulates the expression of many inflammatory mediators and cytokines is activated. The long-term existence of this immune activation can cause brain functions to change, which ultimately leads to types of mental disorders such as anxiety disorder [28]. Furthermore, studies have indicated that the gut microbiota may have an impact on the function of the hypothalamus and pituitary and adrenal glands that can lead to changes in brain functions [29]. Furthermore, an increasing number of basic and clinical studies have shown that the gut microbiota can modulate the communication between the gut and the brain via the gut-brain axis, which mainly includes the nervous system, the immune system and the endocrine system. When the intestinal microbiota is affected, a series of changes in physical or mental symptoms can occur [30-32].

However, it is already known that the gut-brain axis is bidirectional [33]. The integration of the neural complex and hormonal and immune signaling allows for bidirectional communication between the gut and the brain [34] and provides a potential route by which the gut microbiota and its metabolites can access the brain and result in pathophysiological consequences [35]. Communication allows the brain to influence gastrointestinal functions, such as motility, secretion and mucin production [36]. In addition, the imbalance of the hypothalamus and the pituitary and adrenal glands acts as a trigger for inadequate cortisol production and can lead to a condition of dysbiosis with increased production of inflammatory components of the immune system, causing chronic inflammation [37].

\section{Use of prebiotics and probiotics to balance the intestinal microbiota}

According to the World Gastroenterology Organization Global Guidelines (2017) [38], probiotics are defined as "live microorganisms that, when administered in adequate amounts, confer a benefit to the health of the host"; for prebiotics: "a substrate that is selectively used by host microorganisms that confer a health benefit"; and symbiotics: "products that contain probiotics and prebiotics, with health benefits". According to LIU et al. [30], probiotics involve the administration of live microorganisms that must survive the passage of the gastrointestinal tract and have a non-pathogenic characteristic. Prebiotics are also defined by nondigestible food substances that, in the anaerobic fermentation process of intestinal bacteria, bring benefits to the individual through metabolites.

In recent years, the search for alternative methods has increased, seeking to understand the patient as a whole, individualizing their needs and providing health promotion measures, whether through improvement of eating habits, physical activity, mental health, and after detailed analysis of the clinical picture, analyze the possibility of hormone replacement, vitamin and nutrient supplementation in order to provide a better quality of life [39].

\section{Conclusion}

It is essential to seek a healthy lifestyle, aiming at a balance in the metabolism, avoiding the development of inflammatory and immunological diseases such as obesity, hepatic steatosis, mental illnesses including changes in cognition, mood or even even depression. This gear that involves all aspects of human daily life can strongly influence the quality of the intestinal microbiota. Thus, it is important to consider the natural delivery route, longterm breastfeeding, a rational search for healthy eating, stress management, adequate circadian rhythm, physical activity, rational use of medication, avoiding the indiscriminate use of antibiotics, anti-inflammatories and proton pump inhibitors, moderate alcohol use, no smoking and reduced use of xenobiotics. In order to provide a broader treatment, a multidisciplinary follow-up is suggested, including a physician, nutritionist, therapist and physical education professional.

\section{References}

1. Gupta VK, Paul S, Dutta C (2017) Geography, Ethnicity or SubsistenceSpecific Variations in Human Microbiome Composition and Diversity. Frontiers in Microbiology 8: 1162-1178.

2. Donaldson GP, Lee SM, Mazmanian SK (2016) Gut biogeography of the bacterial microbiota. Nature Reviews Microbiology 14(1): 20-32.

3. Marchesi JR, Adams DH, Fava F, Hermes GD, Hirschfield GM, et al. (2016) The gut microbiota and host health: a new clinical frontier. Gut,65(2): 330-339.

4. Danneskiold-Samsøe NB, Barros HDDFQ, Santos R, Bicas JL, Cazarin CBB et al. (2019) Interplay between food and gut microbiota in health and disease. Food research international 115: 23-31.

5. Flint HJ, Duncan SH, Scott KP, Louis P (2015) Links between diet, gut microbiota composition and gut metabolism. Proceedings of the Nutrition Society 74(1): 13-22.

6. Claesson MJ, Cusack S, O'Sullivan O, Greene-Diniz R, de Weerd H, et al. (2011) Composition, variability, and temporal stability of the intestinal microbiota of the elderly. Proceedings of the National Academy of Sciences, 108(Supplement 1): 4586-4591.

7. Toor D, Wsson MK, Kumar P, Karthikeyan G, Kaushik NK, et al. (2019) Dysbiosis disrupts gut immune homeostasis and promotes gastric diseases. International journal of molecular sciences 20(10): 2432.

8. Levy M, Kolodziejczyk AA, Thaiss CA, Elinav E (2017) Dysbiosis and the immune system. Nature Reviews Immunology 17(4): 219-232.

9. Akin H, Tözün N (2014) Diet, microbiota, and colorectal cancer. Journal of clinical gastroenterology 48: S67-S69.

10. Bultman SJ, Interplay between diet, gut microbiota, epigenetic events, and colorectal cancer. Mol Nutr Food Res 2017: 61(1)

11. Barrasa JI, Olmo N, Lizarbe MA, Turnay J (2013) Bile acids in the colon, from healthy to cytotoxic molecules. Toxicology in Vitro 27(2): 964-977.

12. Ramírez-Pérez O, Cruz-Ramón V, Chinchilla-López P, Méndez-Sánchez N (2018) The role of the gut microbiota in bile acid metabolism. Annals of hepatology 16(1): 21-26. 
13. Dvorak K, Payne CM, Chavarria M, Ramsey L, Dvorakova B, et al. (2007) Bile acids in combination with low $\mathrm{pH}$ induce oxidative stress and oxidative DNA damage: relevance to the pathogenesis of Barrett's oesophagus. Gut 56(6): 763-771.

14. Dash S, Clarke G, Berk M, Jacka FN (2015) The gut microbiome and diet in psychiatry: focus on depression. Current opinion in psychiatry 28(1): $1-6$

15. Avershina E, Storrø O, Øien T, Johnsen R, Pope P, et al. (2014) Major faecal microbiota shifts in composition and diversity with age in a geographically restricted cohort of mothers and their children. FEMS microbiology ecology 87(1): 280-290.

16. Le Chatelier E, Nielsen T, Qin J, Prifti E, Hildebrand F, et al. (2013) Richness of human gut microbiome correlates with metabolic markers. Nature 500(7464): 541-546.

17. O’Sullivan O, Cronin O, Clarke SF, Murphy EF, Molloy MG, et al. (2015) Exercise and the microbiota. Gut microbes 6(2): 131-136.

18. Codella R, Luzi L, Terruzzi I (2018) Exercise has the guts: How physical activity may positively modulate gut microbiota in chronic and immunebased diseases. Digestive and Liver Disease 50(4): 331-341.

19. Davis CD (2016) The gut microbiome and its role in obesity. Nutrition today 51(4): 167 .

20. Hsu YJ, Chiu CC, Li YP, Huang WC, Te Huang Y, et al. (2015) Effect of intestinal microbiota on exercise performance in mice. The Journal of Strength \& Conditioning Research 29(2): 552-558.

21. Clarke SF, Murphy EF, O’Sullivan O, Lucey AJ, Humphreys M, et al. (2014) Exercise and associated dietary extremes impact on gut microbial diversity. Gut 63(12): 1913-1920.

22. Yang Z, Scott CA, Mao C, Tang J, Farmer AJ (2014) Resistance exercise versus aerobic exercise for type 2 diabetes: a systematic review and meta-analysis. Sports medicine 44(4): 487-499.

23. Estaki M, Pither J, Baumeister P, Little JP, Gill SK, et al. (2016) Cardiorespiratory fitness as a predictor of intestinal microbial diversity and distinct metagenomic functions. Microbiome 4(1): 1-13.

24. Stewart CJ, Nelson A, Campbell MD, Walker M, Stevenson EJ, et al. (2017) Gut microbiota of type 1 diabetes patients with good glycaemic control and high physical fitness is similar to people without diabetes: an observational study. Diabetic Medicine 34(1): 127-134.

25. Mondin TC, Konradt CE, Cardoso TDA, Quevedo LDA, Jansen K, et al. (2013) Anxiety disorders in young people: a population-based study. Brazilian Journal of Psychiatry 35(4): 347-352.

26. Tomasi J, Lisoway AJ, Zai CC, Harripaul R, Müller DJ, et al. (2019) Towards precision medicine in generalized anxiety disorder: review of genetics and pharmaco (epi) genetics. Journal of psychiatric research 119: 33-47.
27. Toribio-Mateas M (2018) Harnessing the power of microbiome assessment tools as part of neuroprotective nutrition and lifestyle medicine interventions. Microorganisms 6(2): 35.

28. Vaghef-Mehrabany E, Maleki V, Behrooz M, Ranjbar F, EbrahimiMameghani M (2020) Can psychobiotics "mood" ify gut? An update systematic review of randomized controlled trials in healthy and clinical subjects, on anti-depressant effects of probiotics, prebiotics, and synbiotics. Clinical Nutrition 39(5): 1395-1410.

29. Baig D N, Mehnaz S (2021) An Overview of Dairy Microflora. Probiotic Bacteria and Postbiotic Metabolites: Role in Animal and Human Health, pp. 101-137.

30. Liu X, Cao S, Zhang X (2015) Modulation of gut microbiota-brain axis by probiotics, prebiotics, and diet. Journal of agricultural and food chemistry 63(36): 7885-7895.

31. Farzi A, Fröhlich EE, Holzer P (2018) Gut microbiota and the neuroendocrine system. Neurotherapeutics 15(1): 5-22.

32. Petra AI, Panagiotidou S, Hatziagelaki E, Stewart JM, Conti P, et al. (2015) Gut-microbiota-brain axis and its effect on neuropsychiatric disorders with suspected immune dysregulation. Clinical therapeutics 37(5): 984 995.

33. Collins SM, Surette M, Bercik, P (2012) The interplay between the intestinal microbiota and the brain. Nature Reviews Microbiology 10(11): 735-742

34. Mayer EA (2011) Gut feelings: the emerging biology of gut-brain communication. Nature Reviews Neuroscience 12(8): 453-466.

35. Cryan JF, Dinan TG (2012) Mind-altering microorganisms: the impact of the gut microbiota on brain and behaviour. Nature reviews neuroscience 13(10): 701-712

36. Gonzalez A, Stombaugh J, Lozupone C, Turnbaugh PJ, Gordon JI, et al (2011) The mind-body-microbial continuum. Dialogues in clinical neuroscience 13(1): 55 .

37. Mayer EA, Tillisch K, Gupta A (2015) Gut/brain axis and the microbiota. The Journal of clinical investigation 125(3): 926-938.

38. Guarner F, Khan AG, Garisch J, Eliakim R, Gangl A, et al. (2012) World gastroenterology organisation global guidelines: probiotics and prebiotics october 2011. Journal of clinical gastroenterology 46(6): 468481

39. Maiolino ABF, Florencio GP, Saramento T, Silva RR, Brito DVD (2021) Multidisciplinary approach in the treatment of women in climacteria: mind and body connection. SCIOL Biomedicine 4: 170-174. 